An ionization region model for high-power impulse magnetron sputtering discharges

This article has been downloaded from IOPscience. Please scroll down to see the full text article.

2011 Plasma Sources Sci. Technol. 20065007

(http://iopscience.iop.org/0963-0252/20/6/065007)

View the table of contents for this issue, or go to the journal homepage for more

Download details:

IP Address: 130.237.45.197

The article was downloaded on 29/02/2012 at 12:20

Please note that terms and conditions apply. 


\title{
An ionization region model for high-power impulse magnetron sputtering discharges
}

\author{
M A Raadu ${ }^{1}$, I Axnäs ${ }^{1}$, J T Gudmundsson ${ }^{2,3}$, C Huo $^{1}$ and N Brenning ${ }^{1}$ \\ ${ }^{1}$ Division of Space and Plasma Physics, School of Electrical Engineering, Royal Institute of Technology, \\ SE-100 44 Stockholm, Sweden \\ ${ }^{2}$ University of Michigan-Shanghai Jiao Tong University Joint Institute, Shanghai Jiao Tong University, \\ 800 Dong Chuan Road, Shanghai 200240, People's Republic of China \\ ${ }^{3}$ Science Institute, University of Iceland, Dunhaga 3, IS-107 Reykjavik, Iceland \\ E-mail: nils.brenning@ee.kth.se
}

Received 3 July 2011, in final form 10 October 2011

Published 22 November 2011

Online at stacks.iop.org/PSST/20/065007

\begin{abstract}
A time-dependent plasma discharge model has been developed for the ionization region in a high-power impulse magnetron sputtering (HiPIMS) discharge. It provides a flexible modeling tool to explore, e.g., the temporal variations of the ionized fractions of the working gas and the sputtered vapor, the electron density and temperature, and the gas rarefaction and refill processes. A separation is made between aspects that can be followed with a certain precision, based on known data, such as excitation rates, sputtering and secondary emission yield, and aspects that need to be treated as uncertain and defined by assumptions. The input parameters in the model can be changed to fit different specific applications. Examples of such changes are the gas and target material, the electric pulse forms of current and voltage, and the device geometry. A basic version, ionization region model I, using a thermal electron population, singly charged ions, and ion losses by isotropic diffusion is described here. It is fitted to the experimental data from a HiPIMS discharge in argon operated with $100 \mu$ s long pulses and a $15 \mathrm{~cm}$ diameter aluminum target. Already this basic version gives a close fit to the experimentally observed current waveform, and values of electron density $n_{\mathrm{e}}$, the electron temperature $T_{\mathrm{e}}$, the degree of gas rarefaction, and the degree of ionization of the sputtered metal that are consistent with experimental data. We take some selected examples to illustrate how the model can be used to throw light on the internal workings of these discharges: the effect of varying power efficiency, the gas rarefaction and refill during a HiPIMS pulse, and the mechanisms determining the electron temperature.
\end{abstract}

(Some figures may appear in colour only in the online journal)

\section{Introduction}

The magnetron sputtering discharge is a highly successful sputtering technique that has a variety of industrial applications, including protective coatings, optical coatings and thin films for applications in microelectronics. An important development in sputtering technology over the past decade is the high-power impulse magnetron sputtering (HiPIMS) discharge [1-3] which is achieved by applying the power to a conventional magnetron sputtering device in the form of intense short pulses. The result is an increase in plasma density by up to three orders of magnitude $[4,5]$, and a high degree of ionization of the sputtered vapor $[6,7]$ that opens a completely new perspective in the engineering and design of new thin film materials [2]. In the HiPIMS discharge the sputtered flux usually includes more ions than neutral atoms and thus the process can be considered as ionized physical vapor deposition (IPVD) $[2,8,9]$. The possibility to independently vary the pulse length, shape, power and duty cycle opens up new wide ranges of parameters to explore and tune for discharge optimization. Here, we present an ionization region model (IRM) which is intended 


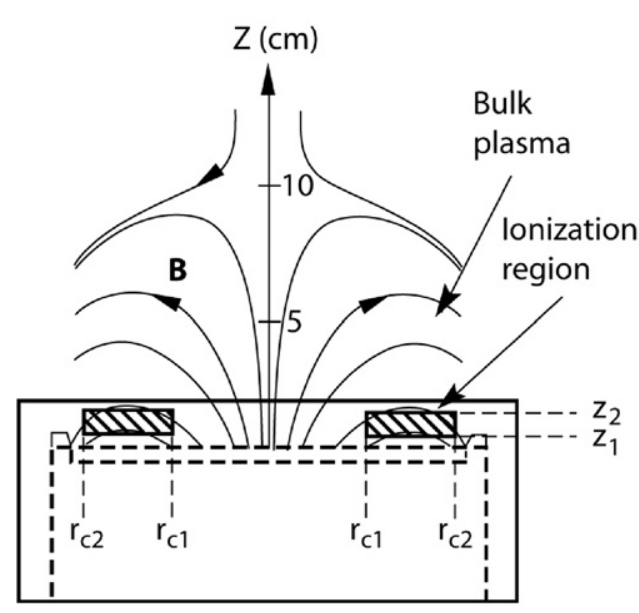

(a)

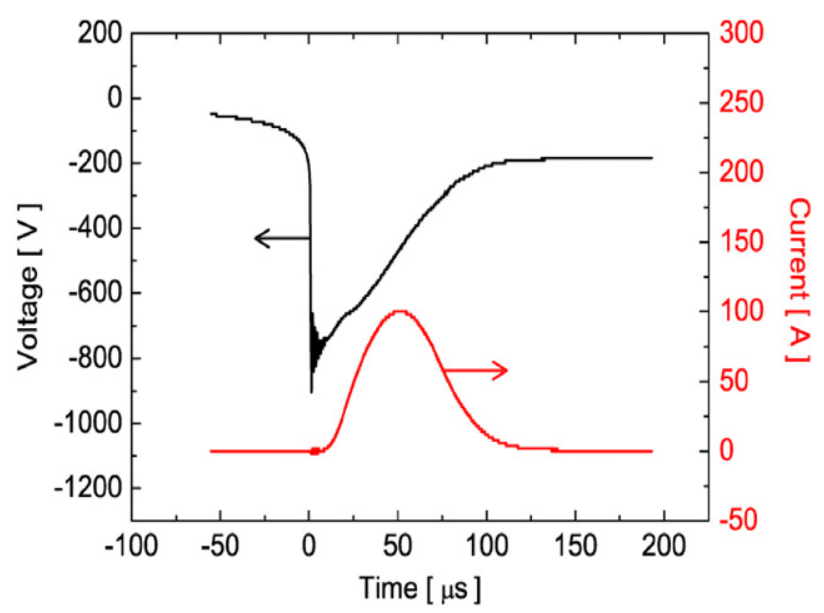

(b)

Figure 1. (a) The magnetic field topology of the HiPIMS discharge at Linköping University, and the definition of the volume covered by the IRM and the BPM of Brenning et al [10]. $(b)$ The measured discharge current $I_{\mathrm{D}}(t)$ and voltage $U_{\mathrm{D}}(t)$ from the pulse that is being modeled.

to be used in this optimization process as a flexible modeling tool. The IRM is based on the fact that in the magnetron sputtering discharge a high density brightly glowing torus shaped plasma hovers and extends a few $\mathrm{cm}$ from the cathode target, and is embedded in a lower density plasma bulk. The IRM is a model for the plasma chemical processes in this high density plasma, while the surrounding plasma is treated in the bulk plasma model (BPM) of Brenning et al [10]. The model can be changed to match different specific applications. Examples of such changes are gas or target material [11], external circuit, geometry, more complex sputtered material dynamics, two-population electron energy distribution functions, and kinetic or thermal gas rarefaction. The basic version IRM I described herein uses the simplest combination of assumptions: a prescribed discharge current and voltage, one thermal electron population, a fixed power fraction to energize the electrons, isotropic particle diffusion, and a simple semi-kinetic (collision/diffusion) treatment of the gas rarefaction process. In a companion paper [12], the model is used for a combined experimental and theoretical study of the reduced deposition rate often found [2] in HiPIMS discharges.

The organization of the paper is as follows. Section 2 contains an overview description of the model and its structure. Details are given in appendices A-C. Section 3 contains model calculations for a particular HiPIMS discharge at Linköping University, with a study of the effects of gas rarefaction, the degree of metal ionization, the degree of self-sputtering, and the electron temperature. Section 4 gives a summary and an overview.

\section{The IRM}

The IRM model is based on the time-dependent global (volume-averaged) model developed by Ashida et al [13] that was later extended to model an IPVD process in an inductively coupled plasma assisted magnetron sputtering discharge by Hopwood [8], and to describe the HiPIMS process by Gudmundsson [14]. The time development is defined by a set of ordinary differential equations giving the first time derivatives of the electron energy (here, the electron temperature) and the particle densities for all the particles. The electron density is directly given by the ion densities assuming quasineutrality of the plasma. The treatment is essentially zero-dimensional, i.e. only volume-averaged values over the whole ionization region (IR) volume for the electron, ion and neutral densities are calculated. However, geometrical effects are included indirectly in the treatment of particle fluxes across the boundaries to the target and the bulk plasma. New features in the IRM compared with the earlier related models [8, 13, 14] are the following.

- A geometric limitation is made to the IR, the smaller part of the discharge vessel in which most of the ionization takes place (see figure 1). The remaining bulk plasma is treated in a separate model [10].

- A fitting parameter $F_{\text {PWR }}$ is introduced, adjustable so that the current to the target calculated in the model best reproduces an experimental discharge current. $F_{\mathrm{PWR}}$ physically represents the fraction of the electric power $U_{\mathrm{D}} I_{\mathrm{D}}(t)$ that goes into heating of the electrons.

- Gas rarefaction [15] is included through a new density balance equation for argon gas atoms.

Figure 2 shows a block diagram illustrating the logical structure of the model. The power block contains the experimentally determined waveforms of the discharge voltage $U_{\mathrm{D}}(t)$ and the discharge current $I_{\mathrm{D}}(t)$, and the fitting parameter $F_{\text {PWR }}$. The core model block contains all mutual interactions between the species through collisions. The treated species are ground state argon atoms Ar (a cold population, plus a hot population due to ions that have recombined at the target), metastable argon atoms $\mathrm{Ar}^{\mathrm{m}}$, argon ions $\mathrm{Ar}^{+}$, metal neutrals $M$ and singly ionized metal ions $M^{+}$. The core block part of the model is the most independent of model assumptions, being based on cross-section data. The 3D aspect block contains the particle fluxes $\Gamma$ of the different species across the boundaries of the IR. These are treated using assumptions about the diffusion and charged particle transport processes, combined with the experimentally based geometry of the IR 


\begin{tabular}{|c|c|c|}
\hline INPUT & CORE MODEL & OUTPUT \\
\hline POWER & \begin{tabular}{|c} 
COUPLED RATE \\
EQUATIONS \\
\end{tabular} & $\begin{array}{c}\text { DENSITIES } \\
\text { TEMPERATURES }\end{array}$ \\
\hline$I_{D}(t), U_{D}(t)$ & Electrons & FLUXES \\
\hline$F_{p w r}$ & $T_{e}, n_{e}$ & $n_{e}(t), T_{e}(t)$ \\
\hline 3-D ASPECTS & $\begin{array}{c}\text { Argon } \\
\text { (working gas) }\end{array}$ & $\begin{array}{c}n_{A r}(t), n_{A r^{\prime \prime}}(t), \\
n_{A r^{+}}(t)\end{array}$ \\
\hline Geometry & $n_{A r}, n_{A r^{m}}$ & \\
\hline$S_{I R}, V_{I R}$ & $n_{A r^{+}}$ & $n_{M}(t), n_{M^{+}}(t)$ \\
\hline Gas rarefaction & \begin{tabular}{|l|} 
Metal (target) \\
\end{tabular} & $\Gamma_{A r}(t), \Gamma_{M}(t)$ \\
\hline$\Gamma_{A r}, \quad \Gamma_{M}$ & $n_{M}, n_{M^{+}}$ & \\
\hline Ion fluxes & & $\Gamma_{A r^{+}}(t), \Gamma_{M^{+}}(t)$ \\
\hline$\Gamma_{A r^{+}}, \Gamma_{M^{+}}$ & & $\underbrace{}_{I_{\text {calc }}(t)}$ \\
\hline
\end{tabular}

Figure 2. A block diagram showing the structure of the IRM.

(approximately the volume with closed magnetic field lines intersecting the target within the racetrack area). The geometry is in this block represented by the surface $S_{\mathrm{IR}}$ and volume $V_{\mathrm{IR}}$. The output block finally contains the densities of all species, the electron temperature, and the fluxes across the boundaries including the calculated discharge current $I_{\text {calc }}$.

\subsection{Electron density and temperature}

The electrons are treated as one population in thermal equilibrium, with an electron temperature $T_{\mathrm{e}}(\mathrm{eV})$. The time rate of change of the electron energy density has three contributions: the externally supplied electrical power input, the surface losses, and the electron energy loss due to inelastic collisions. The temporal development of the electron temperature is followed in time by the rate equation

$$
\begin{aligned}
\frac{3}{2} n_{\mathrm{e}} & \frac{\mathrm{d} T_{\mathrm{e}}}{\mathrm{d} t}=F_{\mathrm{PWR}} \frac{I_{\mathrm{D}} U_{\mathrm{D}}}{e V_{\mathrm{IR}}}-\frac{T_{\mathrm{e}}}{2} \cdot \frac{\left(\Gamma_{\mathrm{Ar}^{+}}+\Gamma_{M^{+}}\right) S_{\mathrm{IR}}}{V_{\mathrm{IR}}} \\
& -\left(E_{\mathrm{Ar}, \mathrm{ci}}+\frac{3}{2} T_{\mathrm{e}}\right) k_{\mathrm{iz}} n_{\mathrm{e}}\left(n_{\mathrm{Ar}}+n_{\mathrm{Ar}, \mathrm{h}}\right) \\
& -\left(E_{\mathrm{m}, \mathrm{ci}}+\frac{3}{2} T_{\mathrm{e}}\right) k_{\mathrm{m}, \mathrm{iz}} n_{\mathrm{e}} n_{\mathrm{Ar}}+E_{\mathrm{dex}} k_{\mathrm{dex}} n_{\mathrm{e}} n_{\mathrm{Ar}^{\mathrm{m}}} \\
& -\left(E_{M, \mathrm{ci}}+\frac{3}{2} T_{\mathrm{e}}\right) k_{M, \mathrm{iz}} n_{\mathrm{e}} n_{M}+\left(6-\frac{3}{2} T_{\mathrm{e}}\right) k_{\mathrm{P}} n_{\mathrm{Ar}^{\mathrm{m}}} n_{M}
\end{aligned}
$$

where $V$ denotes volume, $S$ surface, $\Gamma_{n n}=\left(1 / S_{\mathrm{IR}}\right) \times$ $\int n_{n n} u_{n n} \mathrm{~d} S_{\mathrm{IR}}$ is the averaged flux density of species $n n$ across the boundary, $k=\langle\sigma v\rangle$ is the rate coefficient, and $E$ is the energy in $\mathrm{eV}$. The subscripts denote the following: IR (ionization region), D (discharge), $M$ (metal), $\mathrm{P}$ (Penning ionization), $\mathrm{m}$ (metastable level), iz (ionization), dex (deexcitation), ci (effective cost of ionization, the sum of elastic collisions energy losses per electron-ion pair created), $\mathrm{Ar}$ (cold argon ground state atoms) and Ar,h (hot argon atoms, returning from the target after recombination). The derivation of equation (1) is given in appendix A. The first two terms on the right-hand side correspond to the power block and the $3 \mathrm{D}$ aspect block shown in figure 2 . Since the electron density is determined by quasineutrality, the electron flux across the boundary is obtained from the sum $\left(\Gamma_{\mathrm{Ar}^{+}}+\Gamma_{M^{+}}\right)$of the ion fluxes. The associated energy loss contains work done by the pressure, the convected electron thermal energy, and the energy of ions leaving at the Bohm speed (since their energy is taken from the electrons in the presheath region). For the IRM I, with constant volume and isotropic ion diffusion at the Bohm speed across all surfaces, these terms combine to give the factor $T_{\mathrm{e}} / 2$, see appendix A. The remaining terms in equation (1) describe inelastic collisions. Electron impact ionization produces new electrons that must be heated to the average energy $3 T_{\mathrm{e}} / 2$ and therefore give cooling terms. Penning ionization gives $6 \mathrm{eV}$ energy in excess, but here the new electron also needs $3 T_{\mathrm{e}} / 2$. Electron impact de-excitation $\mathrm{e}+\mathrm{Ar}^{\mathrm{m}} \rightarrow \mathrm{Ar}+\mathrm{e}$ represents an energy gain term for the electrons. The rate coefficients $k\left(T_{\mathrm{e}}\right)$ are taken from the literature and are listed in appendix B.

\subsection{Metal ions and neutrals}

The rate equation for the $M^{+}$ion density is

$\frac{\mathrm{d} n_{M^{+}}}{\mathrm{d} t}=k_{M, \text { iz }} n_{M} n_{\mathrm{e}}+k_{\mathrm{P}} n_{M} n_{\mathrm{Ar}}{ }^{\mathrm{m}}+k_{\mathrm{chexc}} n_{M} n_{\mathrm{Ar}^{+}}-\frac{\Gamma_{M^{+}} S_{\mathrm{IR}}}{V_{\mathrm{IR}}}$.

The first three terms are the internal collision processes in the IR, shown in the left-hand block in figure 3: electron impact ionization, Penning ionization and charge exchange collisions with $\mathrm{Ar}^{+}$ions. The fourth term represents the surface losses. Following Gudmundsson [14], in IRM I the ion fluxes across all surfaces are treated as diffusion to close-lying walls. They are assumed to be isotropic, and at the Bohm speed $u_{\mathrm{B}}$. The single species Bohm criterion is applied separately to each component. A further correction should take into account that there are several ion species, argon and metal ions, but this would require a detailed analysis of the presheath region where the ions are accelerated to satisfy a generalized Bohm criterion. The metal ion flux density is calculated as $\Gamma_{M^{+}}=u_{\mathrm{B}} n_{M^{+}} / 2$. It is reduced by the usual factor $1 / 2$ since only half the particles at the boundary are headed outward. This means that the density at the boundary is taken as equal to half the average density $n_{M^{+}}$. This approximation holds when the mean free path for momentum exchange is larger than the thickness of the IR, $\lambda_{\text {coll }}>L=z_{2}-z_{1}$, which is the case in the runs presented in this paper.

The neutral metal density $n_{M}$ rate equation is

$$
\begin{aligned}
\frac{\mathrm{d} n_{M}}{\mathrm{~d} t} & =-k_{M, \mathrm{iz}} n_{M} n_{\mathrm{e}}-k_{\mathrm{P}} n_{M} n_{\mathrm{Ar}^{\mathrm{m}}}-k_{\mathrm{chexc}} n_{M} n_{\mathrm{Ar}^{+}} \\
+ & \frac{\Gamma_{\mathrm{Ar}^{+}} S_{\mathrm{RT}} Y_{\mathrm{sput}}+\Gamma_{M^{+}} S_{\mathrm{RT}} Y_{\mathrm{self}}}{V_{\mathrm{IR}}}-\frac{\Gamma_{M, \mathrm{diff}}}{L}
\end{aligned}
$$

The three collision terms are familiar from equation (2). The fourth term describes the flux of sputtered metal atoms into the IR which follows from the ion fluxes to the racetrack (with 


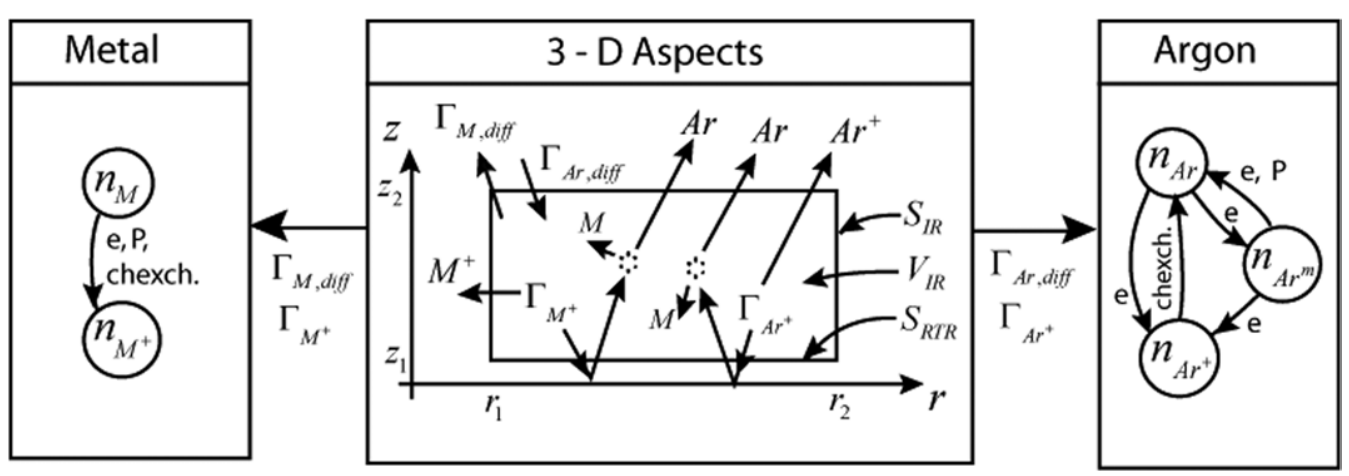

Figure 3. A block diagram illustrating the argon and metal particle balances in IRM I. To the right and left are the collisional processes in the core model block of figure 2 for the metal species and argon species, respectively. The center block contains the 3D aspects that come from the interaction with the surroundings: discharge current, diffusion, sputtering and the gas rarefaction due to the sputter wind. (The minority component of hot argon, returning from recombination at the target (section 2.3), is not shown in the figure.)

the surface area $S_{\mathrm{RT}}$ ), multiplied by the corresponding sputter yields $Y$. The last term in equation (3) describes the diffusion of metal atoms out of the IR. It is given by a flux density at a sputter-equivalent temperature $T_{M}$, but reduced by collisions against argon gas as described in appendix $\mathrm{C}$.

\subsection{Argon neutrals and ions}

The gas rarefaction process is modeled by the time rate of change of the background argon density due to the collision processes in the right-hand block of figure 3, combined with the surface losses (and gains) shown in the central block. The rate equation for the cold argon gas atoms is

$$
\begin{aligned}
\frac{\mathrm{d} n_{\mathrm{Ar}}}{\mathrm{d} t} & =-k_{\mathrm{iz}} n_{\mathrm{e}} n_{\mathrm{Ar}}-k_{\mathrm{exc}, \mathrm{m}} n_{\mathrm{e}} n_{\mathrm{Ar}} \\
+ & k_{\mathrm{chexc}} n_{M} n_{\mathrm{Ar}^{+}}+k_{\mathrm{P}} n_{M} n_{\mathrm{Ar}}+k_{\mathrm{dex}} n_{\mathrm{e}} n_{\mathrm{Ar}}{ }^{\mathrm{m}}+\frac{\Gamma_{\mathrm{Ar}, \mathrm{diff}}}{L} \\
& -\frac{\left(\left(\Gamma_{M, 0}-\Gamma_{M, \mathrm{diff}}\right)+\left(\Gamma_{M^{+}, 0}-\Gamma_{M^{+}, \mathrm{diff}}\right)\right)\left(S_{\mathrm{IR}}-S_{\mathrm{RT}}\right)}{V_{\mathrm{IR}}} \\
& \cdot \frac{m_{M}}{m_{\mathrm{Ar}}} \cdot \frac{n_{\mathrm{Ar}}}{\left(n_{\mathrm{Ar}}+n_{\mathrm{Ar}}\right)} .
\end{aligned}
$$

The terms in the first line describe the collision losses and gains of argon and were discussed in sections 2.1 and 2.2, but the second line requires further comment (this is discussed in more detail in appendix $\mathrm{C}$, where also the variables to be used below are defined). The fourth term of the second line describes the diffusional refill of argon from the surroundings, with a flux density $\Gamma_{\text {Ar,diff }}$ that is determined by the gas temperature and the difference $\left(n_{\mathrm{Ar}, 0}-n_{\mathrm{Ar}}\right)$ in density. The term in the third and fourth line describes the effect of the metal sputter wind on the argon gas, essentially reflecting momentum (or pressure) balance. Due to collisions with argon there is a reduction proportional to $\left(\Gamma_{M, 0}-\Gamma_{M \text {, diff }}\right)+\left(\Gamma_{M^{+}, 0}-\Gamma_{M^{+} \text {,diff }}\right)$ in the metal (both neutral and ions) momentum flow from the IR to the bulk plasma, i.e. across the surface $\left(S_{\mathrm{IR}}-S_{\mathrm{RT}}\right)$. This is balanced here by a corresponding outward argon flux, taking into account the difference in mass, and also the fraction of the lost momentum that goes to metastable argon atoms. Finally we point out that equation (4) has no term for the return flux of recombined $\mathrm{Ar}^{+}$ions from the target. These are treated as a separate hot argon species Ar, h in a simplified treatment, motivated by the fact that this is a minority. The rate equation for $n_{\mathrm{Ar}, \mathrm{h}}$ has a source term and is given by the argon ion flux to the target, taken to return immediately as neutrals with an equivalent temperature of $2 \mathrm{eV}$. (Computer simulations of $\mathrm{Ar}^{+}$surface interaction [16] show that the real energy distribution is far from Maxwellian. For accurate modeling, data are needed concerning the returned energy distribution for each particular target/gas combination used.) The loss terms of $n_{\mathrm{Ar}, \mathrm{h}}$ are diffusion to the bulk plasma, and electron impact ionization.

The rate equations for the metastable argon $\mathrm{Ar}^{\mathrm{m}}$ and ion $\mathrm{Ar}^{+}$densities contain no new physics terms compared with equations (2)-(4). They are therefore not given explicitly.

\subsection{Modeled discharge current}

The discharge current is obtained by considering the fluxes of charged particles just above the racetrack area of the target surface. Since the electrons carry only a small part of the current in the sheath we approximate here the return probability $r$, as defined in [27], of secondary electrons to the target to be zero. This gives

$$
I_{\text {calc }}=e \Gamma_{\mathrm{Ar}^{+}} S_{\mathrm{RT}}\left(1+\gamma_{\mathrm{Ar}^{+}}\right)+e \Gamma_{M^{+}} S_{\mathrm{RT}}\left(1+\gamma_{M^{+}}\right) .
$$

In the IRM I formulation, the model uses a prescribed current $I_{\mathrm{D}}(t)$ in the electron energy balance equation (1). Agreement with equation (5) is obtained by adjusting $F_{\mathrm{PWR}}$. This is necessary to make the model self-consistent from a global energy balance point of view. The obtained value of $F_{\text {PWR }}$ is therefore in practice an output parameter in IRM I, although it is found on the input side in figure 1 .

\section{Results from model runs}

As a model system, data have been taken from a reference discharge in Ar with a $15 \mathrm{~cm}$ diameter $\mathrm{Al}$ target mounted on a slightly unbalanced magnetron. The reason for this choice is that this particular magnetron sputtering discharge has been extensively studied [5-7, 17-22]. It is driven by a Sinex I HiPIMS power supply from Chemfilt Ionsputtering, delivering $100 \mu \mathrm{s}$ long discharge pulses at a repetition frequency of $50 \mathrm{~Hz}$, with a peak current of $100 \mathrm{~A}$, and a peak voltage of 
(a)

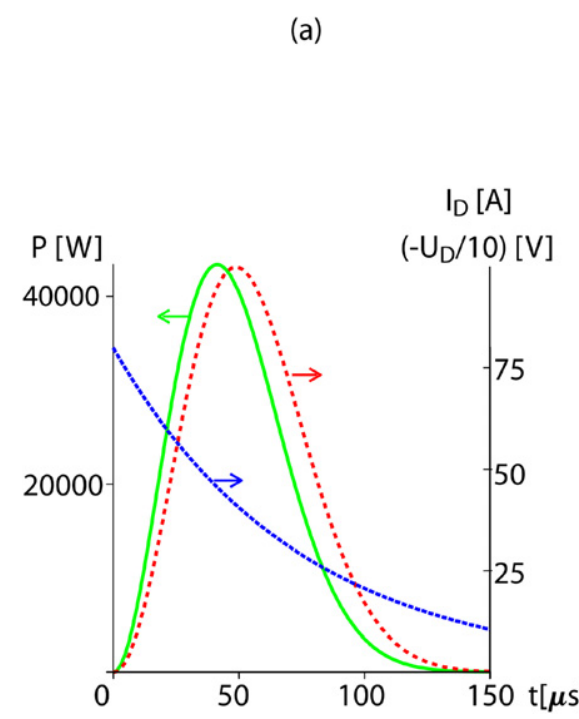

(b)

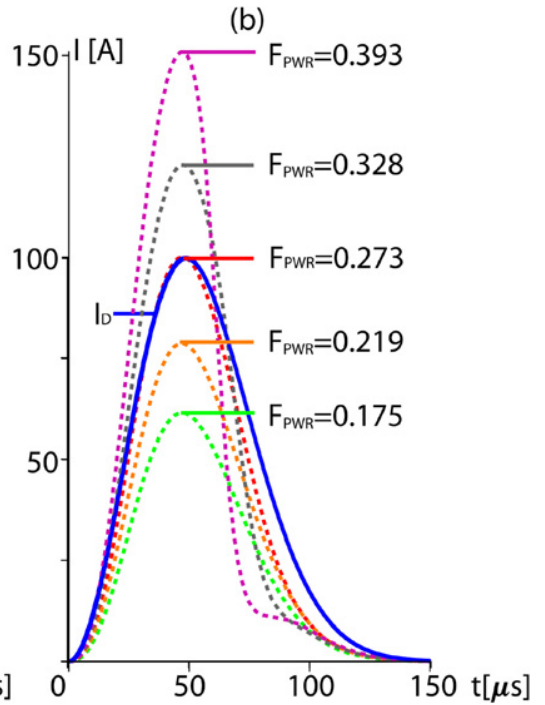

Figure 4. (a) Model input: analytical fits to the experimental HiPIMS pulse shown in figure $1(b)$, of $I_{\mathrm{D}}(t), U_{\mathrm{D}}(t)$, and the electric power $I_{\mathrm{D}}(t) U_{\mathrm{D}}(t)$. (b) Model output: five $I_{\text {calc }}(t)$ curves from equation (5) marked with the used value of the fitting parameter $F_{\mathrm{PWR}}$, which is varied in steps of $20 \%$. The curve with $F_{\mathrm{PWR}}=0.273$ gives a good match to the input current $I_{\mathrm{D}}$.

(a)

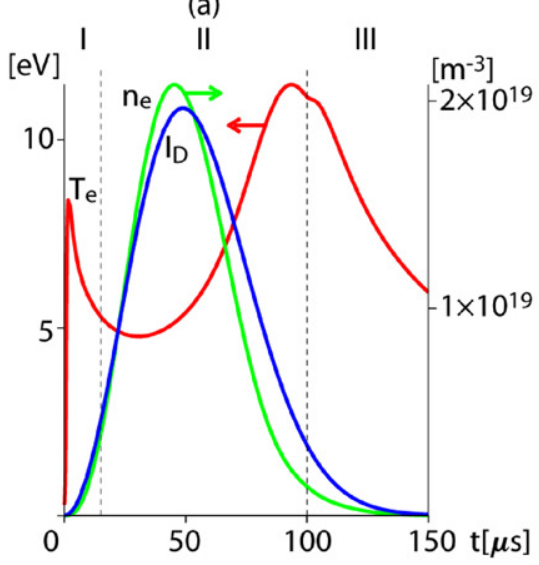

(b)

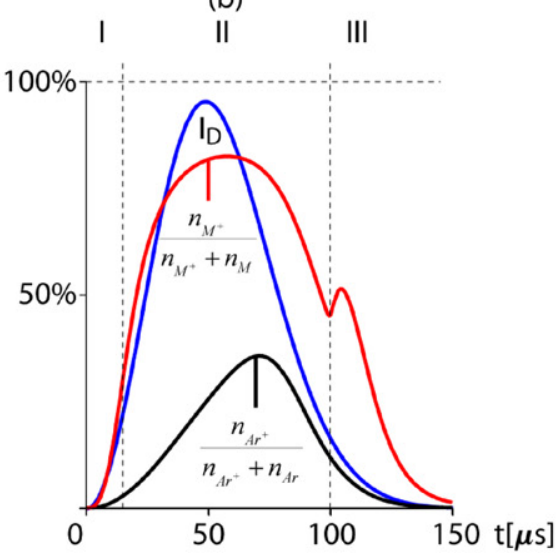

(c)

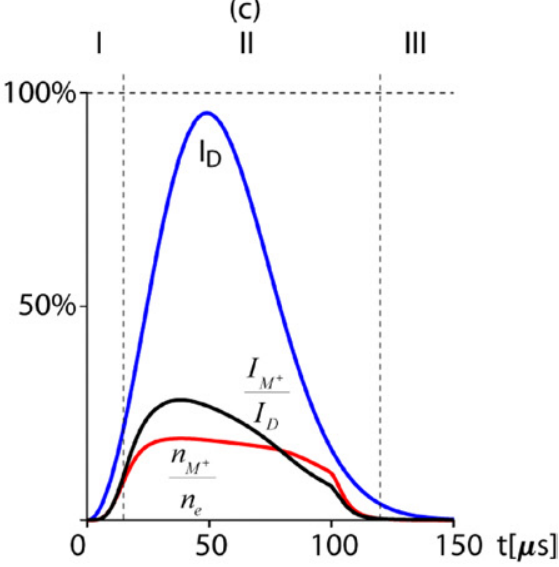

Figure 5. IRM I model results. (a) The plasma density and the electron temperature. (b) The degree of ionization of argon atoms, and of sputtered metal (aluminum) atoms. $(c)$ The fraction of the discharge current to the target that is carried by metal atoms, and the fraction of the ion density that is in the form of metal ions.

$-800 \mathrm{~V}$ which decreases monotonically during the pulse (see figure $1(b))$. The analytical fits used in the model are shown in figure $4(a)$. As standard parameters in the model we use $r_{1}=2 \mathrm{~cm}, r_{2}=7 \mathrm{~cm}$ (based on the racetrack erosion area), $z_{1}=0.1 \mathrm{~cm}$ (based on estimates of the sheath thickness) and $z_{2}=3 \mathrm{~cm}$ (based on plasma density measurements in the same device by Bohlmark et al [17]). Figure 4(b) shows that quite a good current fit is already obtained with a time independent $F_{\mathrm{PWR}}=0.273$. We will therefore use this $F_{\mathrm{PWR}}$ and explore the time development during a HiPIMS pulse in section 3.1, and test the sensitivity to the assumption of the extent in the $z$ direction of the IR in section 3.2.

\subsection{Time development during a HiPIMS pulse}

Figure 5 shows calculated currents, densities and temperatures during a HiPIMS pulse with the fraction of electric power that goes into heating the electrons $F_{\mathrm{PWR}}=0.273$. For time reference, the input discharge current $I_{\mathrm{D}}$ from figure $4(a)$ is plotted in all panels. Different physics dominate in phase I (ignition and breakdown), phase II (main pulse) and phase III (post discharge).

Phase I. Ignition and breakdown, up to $10-15 \mu \mathrm{s}$. The time scale for a uniform plasma to fill the IR can be estimated from the ion travel time as $\tau_{\mathrm{IR}} \approx z_{2} / u_{\mathrm{B}} \approx 0.03 / 3500 \approx 10 \mu \mathrm{s}$, with the Bohm speed $u_{\mathrm{B}}$ calculated for argon ions and with $T_{\mathrm{e}} \approx 5 \mathrm{eV}$ from figure $5(a)$. This time estimate is consistent with experiments $[18,21,23]$ that show rapid changes in this time scale. For times shorter than about $10-15 \mu \mathrm{s}$, the IRM assumption of a constant size of the IR is therefore not valid, and we can only make some qualitative observations. The simulation in figure 5 was started with a seed plasma density $n_{\mathrm{e}}=10^{16} \mathrm{~m}^{-3}$. We have varied the seed in the range $10^{15} \leqslant$ $n_{\mathrm{e}} \leqslant 10^{18}$, and found that before ten microseconds the model has converged in the sense that the result is independent of the seed value. A higher $T_{\mathrm{e}}$ in the beginning of the ignition phase, however, appears in all model runs, independent of the seed 
(a)

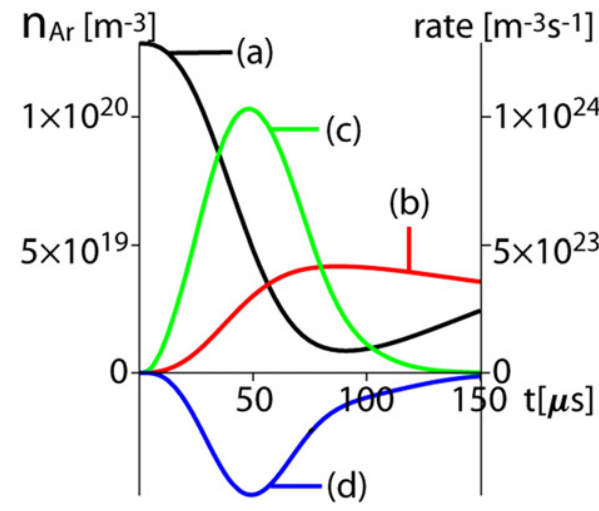

(b)

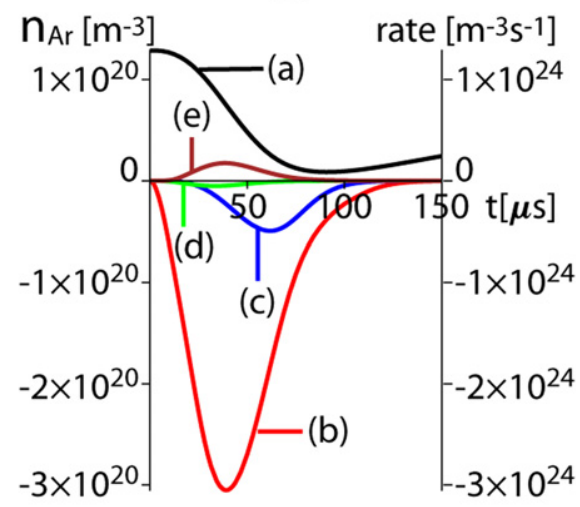

Figure 6. Analysis of the gas rarefaction process. In both panels, curve (a) shows the total argon gas density, i.e. the hot plus cold component. The left panel (a) shows the flux terms involved in the particle balance for argon atoms (equation (4)): Diffusional refill from the bulk plasma, curve (b). Returning Ar, h from the target, curve (c). Escaping Ar, h to the bulk plasma, curve (d). The right panel (b) shows the collision terms in equation (4): The electron impact ionization of cold argon gas, curve (b), and of the hot component, curve (c). The kick-out by the sputter wind, curve $(\mathrm{d})$. The charge exchange term $\times 10$, curve (e).

density. This is consistent with Langmuir probe measurements in our discharge [5,22], and with optical emissions in another HiPIMS device [23] that demonstrate a significant amount of fast electrons during discharge ignition.

Phase II. Main pulse, from $10-15 \mu \mathrm{s}$ up to $100 \mu \mathrm{s}$. The electron density in figure $5(a)$ increases and decays closely in phase with the discharge current. This can be understood as follows. In phase II the two dominating mechanisms for plasma density changes are electron impact ionization inside the IR, and ion loss fluxes across its boundaries. For both processes the density change time constants $\tau_{n_{\mathrm{e}}}=n_{\mathrm{e}} /\left(\mathrm{d} n_{\mathrm{e}} / \mathrm{d} t\right)$ are an order of magnitude below the $100 \mu$ s pulse length. The density during the main pulse is therefore always close to the equilibrium value needed for the ion flux to the racetrack to sustain the momentary value of the current. The peak plasma density is $n_{\mathrm{e}} \approx 2 \times 10^{19} \mathrm{~m}^{-3}$, about twice that reported from the active phase of a pulse, with the same discharge current, in the same device [19].

The degree of argon ionization (figure $5(b)$ ) reaches $36 \%$, and it peaks later than the $I_{\mathrm{D}}$ curve. The reason for this time shift is that, due to extreme gas rarefaction (seen in figure $6(a)$ ), a given argon ion density corresponds to higher degree of ionization at the end of the pulse. The degree of metal ionization (also shown in figure $5(b)$ ) on the other hand has a broader and earlier peak, and a higher degree of ionization, $80-85 \%$. The higher degree of ionization is a direct consequence of the lower ionization potential, $5.96 \mathrm{eV}$ for aluminum compared with $15.76 \mathrm{eV}$ for argon. Electron impact ionization dominates the production of $M^{+}$ions, with the contributions from Penning ionization $\left(M+\mathrm{Ar}^{\mathrm{m}} \rightarrow M^{+}+\right.$ $\mathrm{Ar}+\mathrm{e})$ and charge exchange $\left(M+\mathrm{Ar}^{+} \rightarrow M^{+}+\mathrm{Ar}\right)$ always below $0.35 \%$ and $1.35 \%$, respectively.

The broader and more sustained metal ionization curve shown in figure $5(b)$ can be understood from figure 5(c), which shows the $M^{+}$fraction of the total ion density $\left(n_{M^{+}} /\left(n_{M^{+}}+\right.\right.$ $\left.\left.n_{\mathrm{Ar}^{+}}\right)=n_{M^{+}} / n_{\mathrm{e}}\right)$. It reaches $19 \%$ which is consistent with experiments [6, 7]. $n_{M^{+}}$starts at low values because $n_{M}$ starts at zero, and increases more rapidly than $n_{\mathrm{Ar}^{+}}$due to a combination of decreasing argon density due to gas rarefaction shown in curve (a) of figure $6(a)$, and an increasing accumulated neutral metal density (not shown). After a broad maximum, $n_{M^{+}} / n_{\mathrm{e}}$ decays mainly because the density of sputtered metal from the target decreases toward the end of the pulse, while at $100 \mu \mathrm{s}$ there is a net refill of argon from the bulk plasma region, see curves (a) and (b) in figure $6(a)$.

The fraction $I_{M^{+}} / I_{\mathrm{D}}$ of the discharge current carried by metal ions increases until $40 \mu \mathrm{s}$ and then decreases (see figure $5(c)$ ). The discharge is still far from the regime of sustained self-sputtering (SSS) in which the self-sputtering parameter exceeds unity $\Pi \equiv \alpha \beta Y_{\text {self }}>1[24,25]$, where $\alpha$ is the probability that a sputtered atom is ionized, and $\beta$ is the probability that it after ionization returns to the target. Extracting $\alpha$ and $\beta$ from the model gives an average $\Pi=\alpha \beta Y_{\text {self }} \approx 0.8 \times 0.3 \times 1.1 \approx 0.26$ around the active phase of the pulse.

Gas rarefaction reaches $96.5 \%$, as shown in figure 6, and is significantly delayed in time with respect to the discharge current maximum at $50 \mu \mathrm{s}$. The reason for this delay can be found in the relative sizes, also shown in figure 6 , of the different terms in the argon rate equation (4). The diffusionrefill term has a time constant of several hundred $\mu \mathrm{s}$, and as a consequence the $n_{\mathrm{Ar}}(t)$ curve before about $40-50 \mu \mathrm{s}$ essentially is an integration of the argon loss terms. It has an inflection point close to current maximum where the gas depletion rate is strongest, and a minimum toward the end of the pulse when the sum of the loss terms is balanced by diffusion-refill (curve (b) in figure $6(a)$ ). An interesting observation is that in this HiPIMS discharge the ionization losses (curve (b) in figure 6(b)) dominate over the sputter wind term (curve (d)), a new finding compared with gas rarefaction as earlier modeled in dc magnetron discharges [13] and in Monte Carlo simulations of the HiPIMS discharge [26].

Phase III: Post discharge, after $100 \mu$ s. When the energy input disappears at the end of the pulse, the plasma density in the IR decreases rapidly due to the continued ion loss fluxes (not shown). A different post discharge time evolution of $n_{\mathrm{e}}$ is expected in the surrounding bulk plasma (see figure 1(a)). 
The plasma influx to the bulk plasma from the IR can be extracted as $\left(\Gamma_{M^{+}}+\Gamma_{\mathrm{Ar}^{+}}\right)\left(S_{\mathrm{IR}}-S_{\mathrm{RT}}\right)$ from the $\mathrm{Ar}^{+}$and $M^{+}$ ion density rate equations. It has a peak around the discharge current maximum, and then decays. This is consistent with experimental observation of a plasma pressure wave that is accompanied by a magnetic field perturbation $[5,18]$. This structure is delayed with respect to the discharge current, and moves through the bulk plasma with a velocity of about $1 \mathrm{~km} \mathrm{~s}^{-1}$. Another observation in the post discharge phase is an upward shift in the degree of metal ionization (figure 5(b)). This can be attributed to the fact that no new metal atoms are added by sputtering in this phase, while those that remain in the IR are partially converted to ions by electron impact ionization, Penning ionization and charge exchange collisions $M+\mathrm{Ar}^{+} \rightarrow M^{+}+\mathrm{Ar}$.

The electron temperature increases during the last half of the pulse (figure $5(a)$ ). Various trial model runs with different gas pressures, currents and ion return probabilities show that such electron heating always arises when there is large gas rarefaction, as in figure 6. A simplistic description of the process is that when the argon gas density is lower (and there is insufficient replacement of sputtered metal vapor), the discharge has to adjust by ionizing the remaining gas more rapidly. This simple picture, however, hides a complicated interplay between plasma chemical and discharge physical mechanisms.

\subsection{Variations in the size of the IR}

Hala et al [23] used fast imaging techniques in a HiPIMS discharge similar to ours (unbalanced, with a $100 \mathrm{~mm}$ diameter chromium target) and demonstrated that, after a highly variable ignition phase of 10-20 $\mu$ s, strong light emission is established from a relatively steady region extending $2-3 \mathrm{~cm}$ from the target. The surrounding bulk plasma filled with plasma on a longer time scale, but the light intensity stays an order of magnitude lower, indicating a much lower ionization rate here. The assumed extent of the IR becomes approximately the same if motivated by such observations, or by space- and time-resolved plasma density measurements as by Böhlmark et al [18], and a little larger than the region defined by magnetic field lines that intersect the racetrack part of the target (see figure 1). There is naturally some uncertainty in this choice. We have therefore made test calculations within an estimated range of uncertainty from $z_{2}=0.02$ to $0.04 \mathrm{~m}$. Current matching to $I_{\text {calc }}=I_{\mathrm{D}}$ required adjustments of $F_{\mathrm{PWR}}$, but always less than $20 \%$. The model output data generally only show a little influence from these different assumed $z_{2}$. Some values at current maximum were as follows, listed for calculations using $z_{2}=(0.02,0.03,0.04) \mathrm{m}: F_{\mathrm{PWR}}=(0.228$, $0.273,0.315), T_{\mathrm{e}}=(6.5,5.5,4.9) \mathrm{eV}, n_{\mathrm{e}}=(1.93,2.1,2.15) \times$ $10^{19} \mathrm{~m}^{-3}, n_{M^{+}} / n_{M}=(80,82.5,84) \%$, argon depletion $=$ $(98,96.5,93) \%$, and $n_{\mathrm{Ar}^{+}} / n_{\mathrm{Ar}}=(41,36,31) \%$. These variations are so small that the general conclusions drawn in the preceding section should not be much influenced by the uncertainty in $z_{2}$.

\section{Summary and discussion}

A global plasma chemical model is developed for the ionization region (IR), here defined as the part of magnetron sputtering discharges where most of the ionization takes place. The model, IRM, is constructed so that parameters for different discharge geometries, target materials, pressures, electric pulse forms, and process gases can relatively easily be implemented. It is built on earlier global models $[8,13,14]$ but contains some new features: the introduction of a fitting parameter $F_{\text {PWR }}$ that is adjusted to give electric input power and current that match input experimental data, the introduction of gas rarefaction through a new density balance equation for gas atoms, and a geometric limitation to the ionization region. When the fitting parameter $F_{\mathrm{PWR}}$ is determined by current matching, the model output becomes completely determined by the input experimental data. The surrounding plasma is modeled by a separate bulk plasma model, BPM [10]. In this work, a basic IRM I version is described with one thermal electron population, singly ionized sputtered metal, a collision/diffusion model of gas rarefaction applicable to the low collisionality regime, and isotropic ion losses at the Bohm speed across all boundaries of the ionization region. After adapting the model to a specific HiPIMS discharge, the use of the model is illustrated with a limited exploration of some internal discharge parameters, such as the degrees of metal and argon ionization, the degree of self-sputtering, and the mechanism of gas rarefaction.

The purpose of the IRM model is to provide a semiempirical tool where external discharge parameters (geometry, pressure, pulse form, etc) can be used as input, and conclusions can be drawn regarding internal parameters and processes that are more difficult to measure. Already in its present IRM I form, it has been used for exploration of the ionization fraction of various metals in a HiPIMS discharge by Samuelsson et al [11]. They related this to an increase in the density of the deposited films compared with films grown by a conventional dc magnetron sputtering discharge. A version with variable ion back-attraction probability $\beta$ has been developed and is used to analyze the reduction of deposition rate in HiPIMS in a companion paper (Brenning et al [12]). A third version including two electron populations is used (G Stancu et al 2011, in preparation) to model the complicated development of metastable argon densities during a HiPIMS pulse. The model gives a good reproduction of measurements by laser absorption spectroscopy [44]. Finally, a study of the gas rarefaction during the initial high current peak of long HiPIMS pulses has been performed [45].

\section{Acknowledgments}

This work was financed by the EU FP6 project INNOVATIAL, the Swedish Research Council, and the Icelandic Research Fund. Stimulating discussions with G Stancu, C Vitelaru, T Minea, D Lundin are gratefully acknowledged. Dr K R Lee is thanked for performing simulations to throw light on the energy distribution of returning argon atoms. 


\section{Appendix A. Derivation of the electron power balance equation}

The volume-averaged electron continuity equation can be written in the form

$$
\frac{\mathrm{d} n_{\mathrm{e}}}{\mathrm{d} t}=\Sigma_{\mathrm{e}}-\frac{\Gamma_{\mathrm{e}} S_{\mathrm{IR}}}{V_{\mathrm{IR}}}
$$

where $\Sigma_{\mathrm{e}}$ are the volume-averaged electron sources, and $\Gamma_{\mathrm{e}}$ is the average flux over the surface $S_{\mathrm{IR}}$. SI units are used for energies and temperatures in this appendix. With constant volume there is no work done by the pressure, and the power balance equation per unit volume may be written as $\mathrm{d} W_{\mathrm{e}}=\delta Q_{0}$, a thermodynamic equation where $W_{\mathrm{e}}=(3 / 2) n_{\mathrm{e}} k_{\mathrm{B}} T_{\mathrm{e}}$ is the internal energy density and $\delta Q_{0}$ is the net heating (positive electrical energy input, collisional energy exchange and convected energy losses). The convective energy losses across $S_{\mathrm{IR}}$ are $\Gamma_{\mathrm{e}} S_{\mathrm{IR}}(3 / 2) k_{\mathrm{B}} T_{\mathrm{e}}$ for the electrons. The energy of a cold ion leaving at the Bohm velocity is $(1 / 2) k_{\mathrm{B}} T_{\mathrm{e}}$ [28]. Since the ions are accelerated up to this velocity in the presheath region, this energy must be taken from the electrons, giving a net energy loss $\Gamma_{\mathrm{i}} S_{\mathrm{IR}}(1 / 2) k_{\mathrm{B}} T_{\mathrm{e}}$. Here the ion temperature is neglected since it is taken to be that of the neutral atom and is not associated with an electron energy loss. We define $\delta Q_{1}$ as the net energy loss due to collisions, i.e. without convective losses and electrical heating. Using Kirchhoff's law $\operatorname{div} \boldsymbol{J}=0$ in the form $\Gamma_{\mathrm{i}}=\Gamma_{\mathrm{e}}$, the power balance equation becomes

$$
\begin{aligned}
\mathrm{d} W_{\mathrm{e}} & =-\delta Q_{1}+\frac{F_{\mathrm{PWR}} I_{\mathrm{D}} U_{\mathrm{D}}}{V_{\mathrm{IR}}} \mathrm{d} t \\
& -\left[\frac{3}{2} k_{\mathrm{B}} T_{\mathrm{e}}+\frac{1}{2} k_{\mathrm{B}} T_{\mathrm{e}}\right] \frac{\Gamma_{\mathrm{e}} S_{\mathrm{IR}}}{V_{\mathrm{IR}}} \mathrm{d} t .
\end{aligned}
$$

A more explicit form is

$$
\mathrm{d}\left[\frac{3}{2} n_{\mathrm{e}} k_{\mathrm{B}} T_{\mathrm{e}}\right]=\frac{F_{\mathrm{PWR}} I_{\mathrm{D}} U_{\mathrm{D}}}{V_{\mathrm{IR}}} \mathrm{d} t-\delta Q_{1}-2 k_{\mathrm{B}} T_{\mathrm{e}} \frac{\Gamma_{\mathrm{e}} S_{\mathrm{IR}}}{V_{\mathrm{IR}}} \mathrm{d} t .
$$

This is (in differential form) the equation normally used for calculations with $\delta \mathrm{Q}_{1}$ containing the electron collision terms, and corresponds to that of Gudmundsson [14]. The continuity equation (A1) is used to eliminate the density derivative, giving

$$
\begin{aligned}
n_{\mathrm{e}} d & \left(\frac{3}{2} k_{\mathrm{B}} T_{\mathrm{e}}\right)=\frac{F_{\mathrm{PWR}} I_{\mathrm{D}} U_{\mathrm{D}}}{V_{\mathrm{IR}}} \mathrm{d} t-\left[\delta Q_{1}+\frac{3}{2} k_{\mathrm{B}} T_{\mathrm{e}} \Sigma_{\mathrm{e}} \mathrm{d} t\right] \\
& -\left(\frac{1}{2} k_{\mathrm{B}} T_{\mathrm{e}}\right) \frac{\Gamma_{\mathrm{e}} S_{\mathrm{IR}}}{V_{\mathrm{IR}}} \mathrm{d} t .
\end{aligned}
$$

The loss term $-(3 / 2) k_{\mathrm{B}} T_{\mathrm{e}} \Sigma_{\mathrm{e}} \mathrm{d} t$ is the energy required to heat newly produced electrons to the equilibrium electron temperature. Equation (1) is finally obtained with the use of $\Gamma_{\mathrm{e}}=\Gamma_{\mathrm{i}}=\Gamma_{\mathrm{Ar}^{+}}+\Gamma_{M^{+}}$, introducing the rate coefficients for electron impact and Penning collisions, and breaking up the square bracket into separate collision terms.

\section{Appendix B. Sputtering yields, cross sections and rate coefficients}

For self-sputtering the secondary electron emission yield for metals (clean surface) is given by the semiempirical formula
$[29,30] \gamma_{\mathrm{SSE}}=0.032\left(0.78 E_{\mathrm{iz}}-2 \Phi\right)$, where $E_{\mathrm{iz}}$ is the ionization potential of the metal and $\Phi$ is the work function of the metal surface. For aluminum $\Phi=4.08-4.28 \mathrm{eV}$ and the ionization potential $E_{\mathrm{iz}}=5.96 \mathrm{eV}$ [31]. The process can only occur if $0.78 E_{\mathrm{iz}}>2 \Phi$ so we can assume $\gamma_{M^{+}}=0$ for self-sputtering of and aluminum target in the ion energy range that is of interest here.

For the secondary electron emission yield for argon ions hitting an aluminum target we use the values measured by Yamauchi and Shimizu [32]. A fit to their measured values in the range $700-1500 \mathrm{~V}$ gives $\gamma_{\mathrm{Ar}^{+}}=0.0769+E_{\mathrm{i}} \times 1.1823 \times$ $10^{-5}$, where $E_{\mathrm{i}}$ is the ion bombarding energy in $\mathrm{eV}$, which we extend to zero energy.

The sputtering yields depend on the ion energies which we take, in $\mathrm{eV}$, as the discharge voltage $U_{\mathrm{D}}$. For self-sputtering $\mathrm{Al}^{+} \rightarrow \mathrm{Al}$ we use $Y_{\text {self }}=0.016 E_{\mathrm{Al}^{+}}^{2 / 3}$, based on the data collected by Hayward and Wolter [33] and the calculation by the SRIM code given in [31]. For $\mathrm{Ar}^{+} \rightarrow \mathrm{Al}$ we use the sputtering data collected by Ruzic [34] which up to $1000 \mathrm{eV}$ is well fitted as $Y_{\text {sput }}=2.16 \times 10^{-3} E_{\mathrm{Ar}^{+}}$.

The argon discharge consists of argon atoms in the ground state $\operatorname{Ar}\left(3 s^{2} 3 p^{6}\right)$, metastable argon $\operatorname{Ar}^{\mathrm{m}}$ (the metastable levels $1 \mathrm{~s}^{5}$ and $1 \mathrm{~s}^{3}$ ), and positive argon ions $\mathrm{Ar}^{+}$. In addition we assume metal atoms, $M$, and metal ions, $M^{+}$. Electrons are assumed to have a Maxwellian-energy distribution in the range $1-7 \mathrm{eV}$. The rate coefficients for argon reactions are taken from the literature [8,35-38]. The effective cost of energy $E_{\mathrm{Ar}, \mathrm{ci}}$ per $\mathrm{Ar}^{+}$-e pair created is taken from [38]. The reaction set and rate coefficients for the argon discharge are listed in table 1 . We assume that metal ions are created by electron impact ionization, by Penning ionization, by collisions of metal atoms with electronically excited argon atoms $\left(\mathrm{Ar}^{\mathrm{m}}+M \rightarrow\right.$ $\left.M^{+}+\mathrm{Ar}+\mathrm{e}\right)$ with a rate coefficient $k_{\mathrm{P}}=5.9 \times 10^{-16} \mathrm{~m}^{3} \mathrm{~s}^{-1}$ [39], and through charge exchange $\mathrm{Ar}^{+}+M \rightarrow M^{+}+$Ar with a rate coefficient $k_{\text {chexc }}=1 \times 10^{-15} \mathrm{~m}^{3} \mathrm{~s}^{-1}$ [39]. The rate coefficient for the electron impact ionization of aluminum is calculated from the electron impact ionization cross sections given by Freund et al [40]. The collision energy loss $E_{\mathrm{m} \text {,ci }}$ per electron-ion pair created for the aluminum atom is calculated using the electron impact excitation (to levels $4 \mathrm{~s}, 3 \mathrm{~d}$ and $4 p$ only) and elastic cross sections calculated by Wells and Miller [41].

\section{Appendix C. Collision/diffusion models of the neutral fluxes}

A full treatment of the depletion of the background gas due to collisions with fast neutrals from the cathode would require the modeling of several related processes (among them recombination and re-emission of background gas ions, and sputtering of metal atoms). For example a particlein-cell/Monte Carlo analysis of a dc magnetron sputtering discharge by Kolev and Bogaerts [42] includes neutral plasma particles and self-consistently finds the influence of gas temperature on the discharge behavior. Kadlec [26] has used a Monte Carlo method to simulate the neutral particle flow during a HiPIMS pulse showing a strong rarefaction, heating of the background gas, and the formation of a shock wave. 
Table 1. The reaction set for argon and aluminum. The rate coefficients for electron impact collisions were calculated assuming a Maxwellian electron energy distribution, and fit well over an electron temperature range $1-7 \mathrm{eV}$.

\begin{tabular}{llll}
\hline Reaction & Rate coefficient $\left(\mathrm{m}^{3} \mathrm{~s}^{-1}\right)$ & Threshold $(\mathrm{eV})$ & Reference \\
\hline $\mathrm{e}+\mathrm{Ar} \rightarrow \mathrm{Ar}^{+}+2 \mathrm{e}$ & $k_{\text {iz }}=2.3 \times 10^{-14} T_{\mathrm{e}}^{0.59} \exp \left(17.44 / T_{\mathrm{e}}\right)$ & 15.76 & {$[35]$} \\
$\mathrm{e}+\mathrm{Ar} \rightarrow \mathrm{Ar}^{\mathrm{m}}+\mathrm{e}$ & $k_{\mathrm{ex}}=2.5 \times 10^{-15} \cdot T_{\mathrm{e}}^{0.74} \cdot \exp \left(-11.56 / T_{\mathrm{e}}\right)$ & 11.56 & {$[36]$} \\
$\mathrm{e}+\mathrm{Ar}^{\mathrm{m}} \rightarrow \mathrm{Ar}+\mathrm{e}$ & $k_{\mathrm{dex}}=4.3 \times 10^{-16} \cdot T_{\mathrm{e}}^{0.74}$ & 4.20 & {$[13]$} \\
$\mathrm{e}+\mathrm{Ar}^{\mathrm{m}} \rightarrow \mathrm{Ar}^{+}+2 \mathrm{e}$ & $k_{\mathrm{m}, \mathrm{iz}}=6.8 \times 10^{-15} \cdot T_{\mathrm{e}}^{0.67} \cdot \exp \left(-4.2 / T_{\mathrm{e}}\right)$ & {$[43]$} \\
$\mathrm{e}+\mathrm{Al} \rightarrow \mathrm{Al}^{+}+2 \mathrm{e}$ & $k_{\mathrm{m}, \mathrm{iz}}=1.3467 \times 10^{-13} T_{\mathrm{e}}^{0.3576} \exp \left(-6.7829 / T_{\mathrm{e}}\right)$ & 3.14 & {$[40]$} \\
$\mathrm{e}+\mathrm{Al} \rightarrow \mathrm{Al}(4 \mathrm{~s})+\mathrm{e}$ & $k_{4 \mathrm{~s}}=1.821 \times 10^{-12} T_{\mathrm{e}}^{-0.8679} \exp \left(-6.975 / T_{\mathrm{e}}\right)$ & {$[41]$} \\
$\mathrm{e}+\mathrm{Al} \rightarrow \mathrm{Al}(3 \mathrm{~d})+\mathrm{e}$ & $k_{3 \mathrm{~d}}=5.7148 \times 10^{-12} T_{\mathrm{e}}^{-1.2858} \exp \left(-8.536 / T_{\mathrm{e}}\right)$ & {$[41]$} \\
$\mathrm{e}+\mathrm{Al} \rightarrow \mathrm{Al}(4 \mathrm{p})+\mathrm{e}$ & $k_{4 \mathrm{p}}=1.7195 \times 10^{-12} T_{\mathrm{e}}^{-1.3692} \exp \left(-9.0616 / T_{\mathrm{e}}\right)$ & {$[41]$} \\
$\mathrm{e}+\mathrm{Al} \rightarrow \mathrm{Al}+\mathrm{e}$ & $k_{\mathrm{elast}}=10^{\wedge}\left[-0.0104(\log (E))^{2}+0.1134(\log E)-11.700\right]$ & & {$[41]$} \\
$\mathrm{Ar}+\mathrm{Al} \rightarrow \mathrm{Ar}+\mathrm{Al}^{+}+\mathrm{e}$ & $k_{\mathrm{P}}=5.9 \times 10^{-16}$ & & {$[39]$} \\
$\mathrm{Ar}^{+}+\mathrm{Al} \rightarrow \mathrm{Ar}+\mathrm{Al}^{+}$ & $k_{\text {chexc }}=1.0 \times 10^{-15}$ & {$[39]$} \\
\hline
\end{tabular}

These shock waves have been demonstrated experimentally by Hala et al [23]. These are clearly important processes for the operation of a HiPIMS discharge but are beyond the scope of this work. In IRM I only collisions of argon atoms with fast, sputtered, metal atoms and ions are considered as a first step in the development of a complete model. The analysis uses a cross-section $\sigma_{M, \mathrm{Ar}}=\sigma_{M^{+}, \mathrm{Ar}}$ for a momentum exchange collision (for simplicity assumed to be the same for ground state and metastable argon). The mean free path for a metal atom (or ion) is then $\lambda_{M, \mathrm{Ar}}=1 /\left[\left(n_{\mathrm{Ar}}+n_{\mathrm{Ar}, \mathrm{h}}+n_{\mathrm{Ar}}{ }^{\mathrm{m}}\right) \sigma_{M, \mathrm{Ar}}\right]$. A typical distance through the IR is taken to be $L=z_{2}-z_{1}$. The probability of a collision inside the IR is then $F_{\text {coll }}=$ $1-\exp \left(-L / \lambda_{M, \mathrm{Ar}}\right)$. The loss flux densities in the absence of collisions are $\Gamma_{M, 0}=u_{T_{M}} n_{M} / 2$ and $\Gamma_{M^{+}, 0}=u_{T_{M}} n_{M^{+}} / 2$, with $T_{M}=T_{M^{+}}$and $u_{T_{M}}=\left(2 k_{\mathrm{B}} T_{M} / \pi m_{M}\right)^{1 / 2}$ at a sputterequivalent temperature $T_{M}$. It is assumed here that $\Gamma_{M, 0}$ and $\Gamma_{M^{+}, 0}$ are reduced due to collisions by subtracting the fraction (given by the probability $F_{\text {coll }}$ ) of metal atoms and ions expected to suffer a momentum exchange collision, i.e. $\Gamma_{M, 0}$ is multiplied by a factor $\left(1-F_{\text {coll }}\right)$ giving $\Gamma_{M \text {, diff }}=$ $\Gamma_{M, 0} \exp \left(-L / \lambda_{M, \mathrm{Ar}}\right)$ and $\Gamma_{M^{+}, \mathrm{diff}}=\Gamma_{M^{+}, 0} \exp \left(-L / \lambda_{M, \mathrm{Ar}}\right)$. The momentum lost by the metal atoms and ions is taken up by argon atoms. In each collision they gain a velocity that is greater than the metal atom's velocity loss by the metal to argon mass ratio (from conservation of momentum in a collision). The momentum is shared between ground state and metastable argon atoms in proportion to their relative densities. The consequence for the ground state argon and metastable argon density losses is that an extra loss rate due to kicking out by metal atoms and ions is included. These losses are in equation (4) given by the reduction due to collisions in the metal diffusion loss rate, multiplied by the metal to argon mass ratio and the fractional densities of the ground state and metastable argon atoms, respectively.

The refilling of argon from the background gas is in equation (4) treated as a diffusion process that would exactly balance an argon diffusion loss from the IR for an argon density and temperature equal to those in the background. Here a collisionless limit is used for the diffusion losses assuming that atoms leave the IR with the random flux velocity $u_{\text {ran }}=\sqrt{2 k_{\mathrm{B}} T_{\mathrm{Ar}} / \pi m_{\mathrm{Ar}}}$ across the bounding surfaces. Since by definition only outward moving atoms are involved, the density at the surfaces is taken to be one half of the internal (volume average) density. The loss rate per unit volume is then $n_{\mathrm{Ar}} u_{\mathrm{ran}} S_{\mathrm{IR}} /\left(2 V_{\mathrm{IR}}\right)$. The refill rate, also expressed in terms of unit volume of the IR, therefore (by the requirement of balance for equal external and internal densities) takes the form $n_{\mathrm{Ar} 0} u_{\mathrm{ran}} S_{\mathrm{IR}} /\left(2 V_{\mathrm{IR}}\right)$ where $n_{\mathrm{Ar} 0}$ is the density of the (external) background gas. The random flux velocities would be different if the heating of the argon gas within the IR is taken into account. A more detailed modeling of the diffusion losses including collisions, and the geometry of the IR, as in the case of the Knudsen diffusion of metals in cylindrical geometry discussed elsewhere [8,14], is possible but the present analysis is used here as a reasonable first approach for the parameter range $\lambda_{\text {coll }}>L$, which obtains for the experiment that is modeled in this paper.

\section{References}

[1] Kouznetsov V, Macák K, Schneider J M, Helmersson U and Petrov I 1999 A novel pulsed magnetron sputter technique utilizing very high target power densities Surf. Coat. Technol. 122290

[2] Helmersson U, Latteman M, Bohlmark J, Ehiasaran A P and Gudmundsson J T 2006 Ionized physical vapor deposition (IPVD): a review of technology and applications Thin Solid Films 5131

[3] Sarakinos K, Alami J and Konstantinidis S 2010 High power pulsed magnetron sputtering: a review on scientific and engineering state of the art Surf. Coat. Technol. 2041661

[4] Ehiasarian A P, New R, Münz W D, Hultman L, Helmersson U and Kouznetsov V 2002 Influence of high power densities on the composition of pulsed magnetron plasmas Vacuum 65147

[5] Gudmundsson J T, Alami J and Helmersson U 2002 Spatial and temporal behavior of the plasma parameters in a pulsed magnetron discharge Surf. Coat. Technol. 161249

[6] Bohlmark J, Lattemann M, Gudmundsson J T, Ehiasarian A P, Aranda Gonzalvo Y, Brenning N and Helmersson U 2006 The ion energy distributions and ion flux composition from a high power impulse magnetron sputtering discharge Thin Solid Films $\mathbf{5 1 5} 1522$

[7] Bohlmark J, Alami J., Christou C, Ehiasarian A P and Helmersson U 2005 Ionization of sputtered metal in high power impulse magnetron sputtering J. Vac. Sci. Technol. A 2318

[8] Hopwood J A 2000 Plasma physics Thin Films: Ionized Physical Vapor Deposition ed J A Hopwood (San Diego, CA: Academic) p 181 
[9] Gudmundsson J T 2010 The high power impulse magnetron sputtering discharge as an ionized physical vapor deposition tool Vacuum 841360

[10] Brenning N, Axnäs I, Raadu M A , Lundin D and Helmerson U 2008 A bulk plasma model for $\mathrm{dc}$ and HiPIMS magnetrons Plasma Sources Sci. Technol. 17045009

[11] Samuelsson M, Lundin D, Jensen J, Raadu M A, Gudmundsson J T and Helmersson U 2010 On the film density using high power impulse magnetron sputtering Surf. Coat. Technol. 205591

[12] Brenning N, Huo C, Lundin, D, Raadu M A, Vitelaru C, Stancu G D, Minea T and Helmersson U 2010 Understanding deposition rate loss in high power impulse magnetron sputtering Plasma Sources Sci. Technol. submitted

[13] Ashida S, Lee C and Lieberman M A 1995 Spatially averaged (global) model of time modulated high density argon plasmas J. Vac. Sci. Technol. A 132498

[14] Gudmundsson J T 2008 Ionization mechanism in the high power impulse magnetron sputtering (HiPIMS) discharge J. Phys.: Conf. Ser. 100082013

[15] Rossnagel S M 1988 Gas density reduction effects in magnetrons J. Vac. Sci. Technol. A 619

[16] Lee K-R 2010 private communication

[17] Bohlmark J, Helmersson U, VanZeeland M, Axnäs I, Alami J and Brenning N 2004 Measurements of the magnetic field change in a pulsed high current magnetron discharge Plasma Sources Sci. Technol. 13654

[18] Bohlmark J, Gudmundsson J T, Alami J, Lattemann M and Helmersson U 2005 Spatial electron density distribution in a high-power pulsed magnetron discharge IEEE Trans. Plasma Sci. 33346

[19] Lundin D, Helmersson U, Kirkpatrick S, Rohde S and Brenning N 2008 Anomalous electron transport in high power impulse magnetron sputtering Plasma Sources Sci. Technol. 17025007

[20] Lundin D, Larsson P, Wallin E, Lattemann M, Brenning N and Helmersson U 2008 Cross-field ion transport during high power impulse magnetron sputtering Plasma Sources Sci. Technol. 17035021

[21] Lundin D 2010 PhD Thesis: The HiPIMS Process, Linköping Studies in Science and Technology, Dissertation No 1305, Linköping, Sweden

[22] Gudmundsson J T, Sigurjonsson P, Larsson P, Lundin D and Helmerson U 2009 On the electron energy in the high power impulse magnetron sputtering discharge J. Appl. Phys. 105123302

[23] Hala M, Viau N, Zabeida O, Klemberg-Sapieha J E and Martinu L 2010 Dynamics of reactive high-power impulse magnetron sputtering discharge studied by time- and space-resolved optical emission spectroscopy and fast imaging J. Appl. Phys. 107043305

[24] Hosokawa N, Tsukada T and Kitahara H 1980 Proc. 8th Int. Vacuum Congress (Cannes, France, 22-26 September 1980) p 11

[25] Andersson J and Anders A 2009 Self-sputtering far above the runaway threshold: an extraordinary metal-ion generator Phys. Rev. Lett. 102045003

[26] Kadlec S 2007 Simulation of neutral particle flow during high power magnetron impulse Plasma Process. Polym. 4 S419
[27] Vlcek J and Burcalova K 2010 A phenomenological equilibrium model applicable to high-power pulsed magnetron sputtering Plasma Sources Sci. Technol. 19065010

[28] Chen F F 1985 Introduction to Plasma Physics and Controlled Fusion vol 1, 2nd edn (New York: Plenum) p 293

[29] Baragiola R A, Alonso E V, Ferron J and Oliva-Florio A 1979 Ion induced electron emission from clean metal Surf. Sci. 90240

[30] Baragiola R A and Riccardi P 2008 Electron emission from surfaces induced by slow ions and atoms Reactive Sputter Deposition ed D Depla and S Mahieu (Berlin: Springer) pp 43-60

[31] Anders A, Andersson J and Ehiasarian A 2007 High power impulse magnetron sputtering: Current-voltage-time characteristics indicate the onset of sustained self-sputtering J. Appl. Phys. 102113303

[32] Yamauchi Y and Shimizu R 1983 Secondary electron emission from aluminum by argon and oxygen ion bombardment below $3 \mathrm{keV}$ Japan. J. Appl. Phys. 22 L227

[33] Hayward W H and Wolter A R 1969 J. Appl. Phys. 402911

[34] Ruzic D N 1990 Handbook of Plasma Processing Technology: Fundamentals, Etching, Deposition, and Surface Engineering ed S M Rossnagel et al (Park Ridge, NJ: Noyes Publications) pp 70-90

[35] Gudmundsson J T and Thorsteinsson E G 2007 Oxygen discharges diluted with argon: dissociation processes Plasma Sources Sci. Technol. 16399

[36] Lee M-H and Chung C-W 2005 Self-consistent global model with multi-step ionizations in inductively coupled plasmas Phys. Plasmas 12073501

[37] Lee C and Lieberman M A 1995 Global model of $\mathrm{Ar}, \mathrm{O}_{2}, \mathrm{Cl}_{2}$, and $\mathrm{Ar} / \mathrm{O}_{2}$ high-density plasma discharges $J$. Vac. Sci. Technol. A 13368

[38] Lieberman M A and Lichtenberg A J 2005 Principles of Plasma Discharges and Materials Processing 2nd edn (New York: Wiley) p 81

[39] Lu J and Kushner M J 2000 Effect of sputter heating in ionized metal physical vapor deposition reactors J. Appl. Phys. 877198

[40] Freund R S, Wetzel R C, Shul R J and Hayes T R 1990 Cross-section measurements for electron-impact ionization of atoms Phys. Rev. A 413575

[41] Wells C A and Miller K J 1975 Effect of a superposition of configurations on the generalized oscillator strengths and elastic and inelastic total cross sections for the $3 p$ to $4 s, 4 p$, and nd $(n \leqslant 7)$ transitions in aluminum Phys. Rev. A 1217

[42] Kolev I and Bogaerts A 2008 Calculation of gas heating in a dc sputter magnetron J. Appl. Phys. 104093301

[43] Kannari F, Obara M and Fujioka T 1985 An advanced kinetic model of electron-beam-excited $\mathrm{KrF}$ lasers including the vibrational relaxation in $\mathrm{KrF}^{*}(\mathrm{~B})$ and collisional mixing of $\mathrm{KrF}^{*}(\mathrm{~B}, \mathrm{C})$ J. Appl. Phys. 574309

[44] Vitelaru C, Lundin D, Stancu G D, Brenning N, Bretagne J and Minea T 2011 Argon metastables in HiPIMS. Time-resolved tunable diode laser diagnostics Plasma Sources Sci. Technol. submitted

[45] Huo C, Raadu, M A, Lundin D, Gudmundsson J T, Anders A and Brenning N 2011 Gas rarefaction and the time evolution of long HiPIMS pulses Plasma Sources Sci. Technol. submitted 